\title{
Article
}

\section{Experimental and Computational Evidence of the Biradical Structure and Reactivity of Titanium(IV) Enolates}

Carlos Heras, Alejandro Gómez-Palomino, Pedro Romea, Fèlix Urpí, Josep Maria Bofill, and Ibério de P.R. Moreira

J. Org. Chem., Just Accepted Manuscript • DOI: 10.1021/acs.joc.7b01174 • Publication Date (Web): 07 Aug 2017

Downloaded from http://pubs.acs.org on August 15, 2017

\section{Just Accepted}

"Just Accepted" manuscripts have been peer-reviewed and accepted for publication. They are posted online prior to technical editing, formatting for publication and author proofing. The American Chemical Society provides "Just Accepted" as a free service to the research community to expedite the dissemination of scientific material as soon as possible after acceptance. "Just Accepted" manuscripts appear in full in PDF format accompanied by an HTML abstract. "Just Accepted" manuscripts have been fully peer reviewed, but should not be considered the official version of record. They are accessible to all readers and citable by the Digital Object Identifier (DOI®). "Just Accepted" is an optional service offered to authors. Therefore, the "Just Accepted" Web site may not include all articles that will be published in the journal. After a manuscript is technically edited and formatted, it will be removed from the "Just Accepted" Web site and published as an ASAP article. Note that technical editing may introduce minor changes to the manuscript text and/or graphics which could affect content, and all legal disclaimers and ethical guidelines that apply to the journal pertain. ACS cannot be held responsible for errors or consequences arising from the use of information contained in these "Just Accepted" manuscripts. 


\title{
Experimental and Computational Evidence of the
}

\section{Biradical Structure and Reactivity of Titanium(IV)}

\author{
Enolates

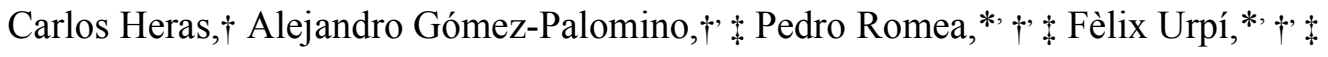 \\ Josep Maria Bofill, ${ }^{*}, \uparrow \S$ and Ibério de P. R. Moreira*, \# $\S$ \\ $\dagger$ Departament de Química Inorgànica i Orgànica, Secció de Química Orgànica. Universitat de Barcelona, \\ Carrer Martí i Franqués 1-11, 08028 Barcelona, Catalonia, Spain. \\ $\$$ Institut de Biomedicina (IBUB), Universitat de Barcelona. \\ § Institut de Quimica Teòrica i Computacional de la Universitat de Barcelona (IQTCUB) \\ \# Departament de Ciència dels Materials i Química Física, Secció de Química Física, Universitat de Barcelona \\ Carrer Martí i Franqués 1-11, 08028 Barcelona, Catalonia, Spain \\ E-mail: i.moreira@ub.edu
}

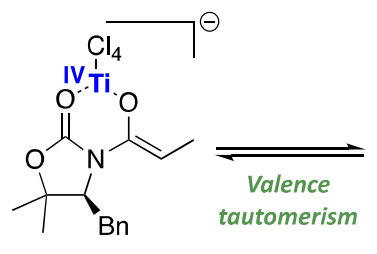

Nucleophile
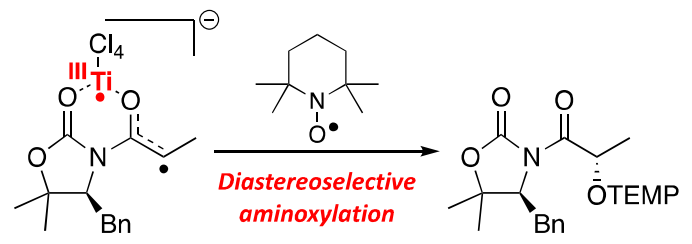

Biradical

\begin{abstract}
Quantum chemical calculations have unveiled the unexpected biradical character of titanium(IV) enolates from $\mathrm{N}$-acyl oxazolidinones and thiazolidinethiones. The electronic structure of these species therefore involves a valence tautomerism consisting of an equilibrium between a closed shell (formally Ti(IV) enolates) and an open shell, biradical, singlet (formally $\mathrm{Ti}$ (III) enolates) electronic states, whose origin is to be basically found in changes of the $\mathrm{Ti}-\mathrm{O}$ distance. Spectroscopic studies of the intermediate species lend support to such a model, which also turns out to be crucial for a better understanding of the overall reactivity of titanium(IV) enolates. In this context, a thorough computational analysis of the
\end{abstract}


radical addition of titanium(IV) enolates from $N$-acyl oxazolidinones to TEMPO has permitted us to suggest an entire mechanism, which accounts for the experimental details and the diastereoselectivity of the process. All together, this evidence highlights the relevance of biradical intermediates from titanium(IV) enolates and may be a useful contribution to the foundations of a more insightful comprehension of the structure and reactivity of titanium(IV) enolates.

\section{INTRODUCTION}

Metal enolates play a crucial role in a wide range of carbon-carbon as well as carbonheteroatom bond forming reactions and are therefore among the most important nucleophilic species in organic synthesis. ${ }^{1-3}$ Alkylation or aldol reactions are well-known examples of the sort of transformations that hinge on the enolization of a parent carbonyl compound and the reactivity of the resultant enolate. ${ }^{3-5}$ Therefore, great effort has been dedicated to the development of increasingly more efficient enolization procedures that supply metal enolates capable of participating in highly selective reactions. ${ }^{6}$ As a result of such research, a handful of procedures have emerged as the most appropriate to obtain metal enolates with remarkable regio- and stereocontrol, which can subsequently react with a broad range of electrophiles under a variety of mild conditions. ${ }^{7}$ A proper understanding of these processes is thus fundamental to appreciate the prominent role of metal enolates as nucleophilic reagents in organic transformations that are governed by heterolytic mechanisms.

In such a scenario, the metal counterpart is only required to provide oxygen-bound metal enolates with a defined geometry that interact with electrophiles through tight and ordered transition states. Therefore, the importance of the metal depends on its influence on both the structure and the reactivity of the organic part of the enolate. The alternative point of view 
concerning the inverse effect of the organic part of the enolate on the metal has hardly been considered. Nevertheless, such a perspective could reveal new forms of reactivity that are complementary to the aforementioned nucleophilic pathway.

The non-innocent ligand concept, expounded by Jørgensen in the $1960 \mathrm{~s},{ }^{8}$ can be useful to address such a shift in the reacting paradigm. The concept applies to those ligands that can alter the oxidation state of the metal, which dramatically affects the reactivity of the overall organometallic species. ${ }^{9,10}$ Unfortunately, the theoretical background for such a phenomenon is often misunderstood since it can imply either a resonance case for a single minimum or a real equilibrium between two different species in a double minimum arrangement. The former corresponds to a hybrid valence bond electronic state of a given molecular structure, while the latter properly corresponds to a valence tautomery. ${ }^{11}$ Irrespective of the theoretical model that best fits this situation, the main consequence of this new approach is that the metal and the ligand can act in a synergistic manner thereby enabling new chemical transformations to occur.

Within this framework, some years ago we reported the unexpected biradical character of titanium(IV) enolates derived from $\alpha$-benzyloxy ketones ${ }^{12}$ and $\mathrm{TiCl}_{4}$-phenoxy complexes ${ }^{13}$ that were assigned to coexisting Ti(III) species. Rather than being a simple expansion of the resonance model, this biradical character arises from a valence tautomery in which there is a nuclear configuration with two utterly unlike but almost degenerate electronic configurations. Indeed, the very nature of these electronic configurations is, to a large extent, distinct: one corresponds to a closed shell electronic state, whereas the other has a marked open shell, delocalized biradical, character, which requires an electron transfer from the organic ligand to the titanium metal. Therefore, the closed shell electronic configuration would be responsible for the classical nucleophilic reactivity observed for these titanium(IV) enolates ${ }^{14}$ whereas the open shell might be the origin of the aforementioned biradical character and the basis for a 
new reacting paradigm: titanium(IV) enolates can also participate in homolytic or radical additions (Scheme 1).

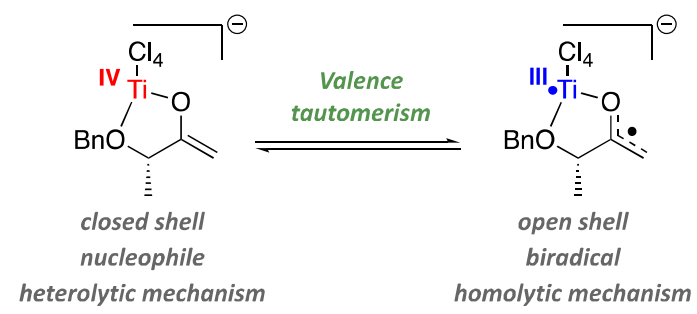

Scheme 1. Valence tautomerism in titanium(IV) enolates ${ }^{12}$

Hence, the reactivity of titanium(IV) enolates would follow different lines if their organic part acted as a non-innocent ligand and thus affected the oxidation state of the metal. The interaction of such a ligand with the metal might dramatically change the electronic distribution of the ligand itself and activate new and unexpected reactivity of it. Homocoupling of certain enolates derived from phenylacetic derivatives ${ }^{15,16}$ and other occasional pieces of evidence in the literature ${ }^{17}$ had already suggested the feasibility of such a new reaction mode. Interestingly, Zakarian recently proved that titanium(IV) enolates derived from chiral $N$-acyl oxazolidinones could indeed react stereoselectively with carbon- as well as oxygen-based radicals through a homolytic mechanism (Equation 1 and 2 in Scheme 2). ${ }^{18-20}$ 
(1)

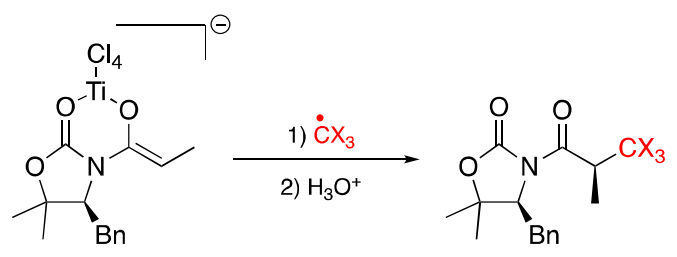

(2)

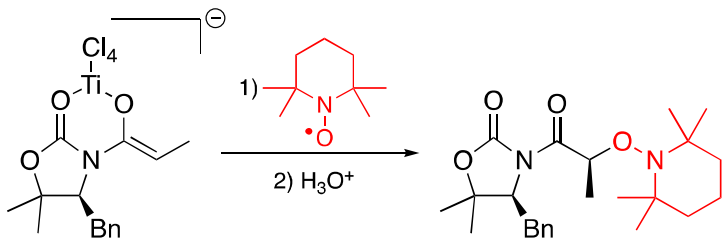

(3)
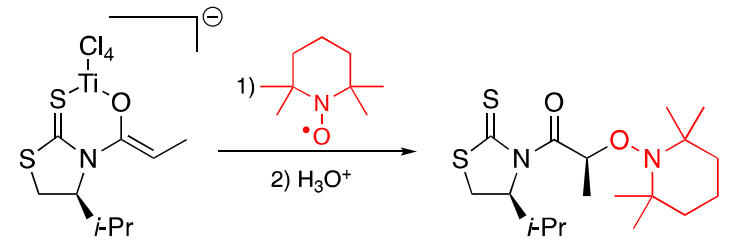

Scheme 2. Stereoselective reactions of titanium(IV) enolates with radicals ${ }^{18-21}$

Furthermore, we also fully reported the aminoxylation reactions of titanium(IV) enolates from several chiral platforms with TEMPO that produce $\alpha$-oxygenated adducts in a highly stereocontrolled manner compatible with a biradical intermediate (Equation 2 and 3 in Scheme 2). ${ }^{21}$ Thus, the valence tautomery in titanium(IV) enolates shown in Schemes 1 and 2 is well established, which is also compatible with the observed nucleophilic-like reactivity. ${ }^{14}$ However and despite the success of such transformations, the origin of the biradical character of the titanium(IV) enolates remained elusive. Thus, running parallel to our studies of the reactivity of titanium(IV) enolates from a wide range of $N$-acyl oxazolidinones and thiazolidinethiones, we carried out a comprehensive theoretical analysis of the valence tautomery equilibrium as well as the mechanism of their aminoxylation reaction with TEMPO. Herein, we report our findings concerning the origin of the valence tautomery in the titanium(IV) enolates from $N$-propanoyl oxazolidinone $\mathbf{1}$ and thiazolidinone $\mathbf{2}$ represented in Figure $1 .^{22}$

Our calculations on their electronic structure have established that the biradical character of these titanium(IV) enolates stems from changes in the geometry of both species. Therefore, 
nucleophilic titanium(IV) enolates are associated with biradical Ti(III) species through a valence tautomery equilibrium, which basically hinges on differences of Ti-O bond distance in the case of oxazolidinones, and a more complex distortion of the $\mathrm{Ti}-\mathrm{S}$ bond in the case of thiazolidinethiones. Further studies have also revealed that a radical-like mechanism successfully accounts for the reaction of titanium(IV) enolates from 1 with TEMPO. Importantly, these calculations account for the diastereoselectivity observed in the aminoxylation reactions shown in Scheme 2. All together, these results strongly support the new reacting paradigm of titanium(IV) enolates. 


\section{RESULTS AND DISCUSSION}

\section{Analysis of the valence tautomerism in the titanium(IV) enolates}

With the aim of providing a theoretical account of the valence tautomerism associated with the titanium(IV) enolates described in Scheme 2, we initially studied the electronic and molecular structures of simple chiral models $\mathbf{1}$ and $\mathbf{2}$ in detail (Figure 1).
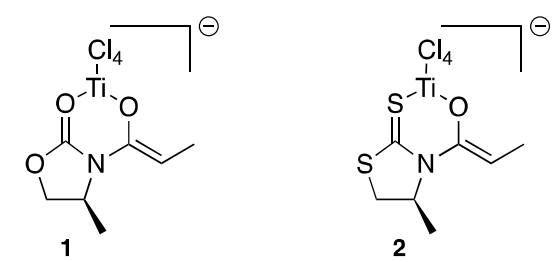

Figure 1. Models used to investigate the valence tautomerism of the titanium(IV) enolates reported in this work.

The molecular structure of titanium enolate $\mathbf{1}$ features an almost planar chelate with four chlorine atoms directly bound to the hexacoordinated titanium, which can be properly described by means of a closed shell electronic configuration. Nevertheless, a high polarization of the electron density of the $\mathrm{TiCl}_{4}$ moiety towards the chlorine atoms and the availability of empty d-orbitals for the metal facilitated open shell configurations. Indeed, our calculations identified low energy open shell singlet (OSS) and triplet (T) electronic states with a very strong biradical character in which one electron is mainly located over the titanium atom and the other one forms an allylic-like system $\left[\mathrm{O}-\mathrm{CR}-\mathrm{CH}_{2}\right] \bullet$ Such a species might be classified as a titanium(III) enolate. Our results indicate that this is close in energy to the titanium(IV) enolate, and these two species are connected through a reaction path with a low energy barrier. Analysis of Ti-O bonds both in closed- and open-shell configurations also 
suggests that these interactions are dative in nature. The most relevant valence (natural) orbitals of this biradical, along with the corresponding occupation numbers are shown in Figure 2.

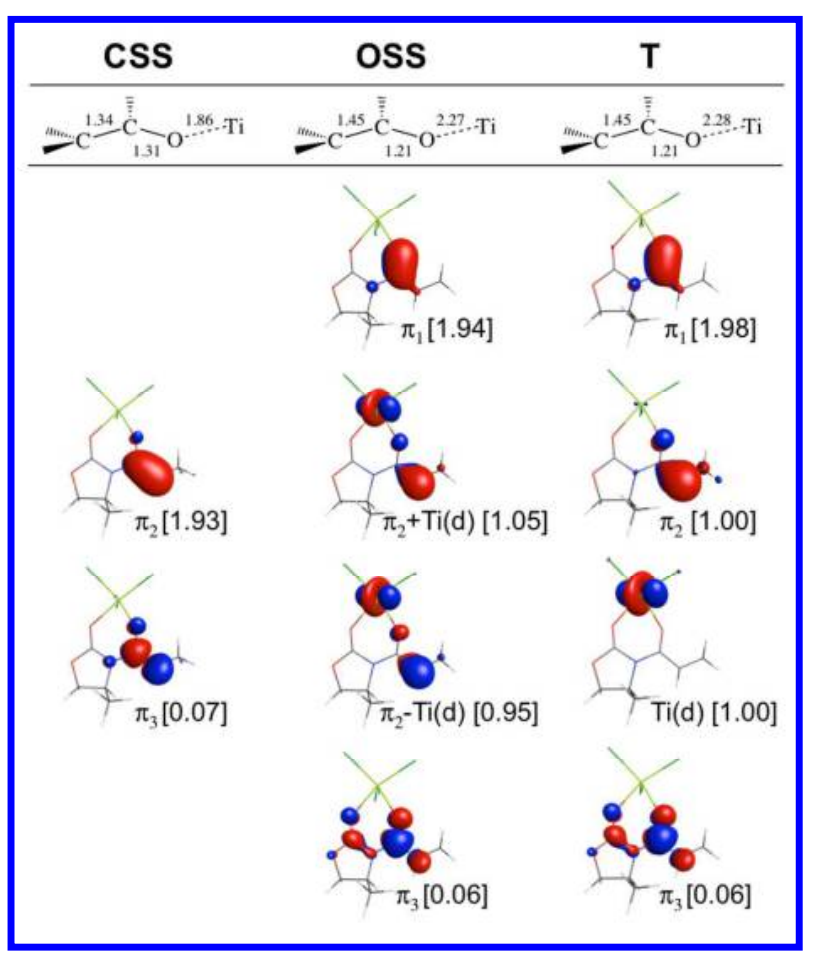

Figure 2. Relevant $\operatorname{CASSCF}(8,7)$ natural orbitals of the electronic configurations of $\mathbf{1}$ and selected distances of optimized structures. Values in square brackets are the corresponding occupation numbers.

The topology of these orbitals allows us to assign these orbitals to an allylic-like $\pi$-system $\left(\pi_{1}\right.$, $\pi_{2}$, and $\pi_{3}$ orbitals) combined with the Ti d orbital, Ti(d). Orbitals $\pi_{1}$ and $\pi_{3}$ involve the bonding and antibonding combinations of the allyl-like $\pi$-system. In turn, the non-bonding $\pi_{2}$ and $\operatorname{Ti}(d)$ orbitals can mix to produce orbitals $\pi_{2}+\operatorname{Ti}(d)$ and $\pi_{2}-\mathrm{Ti}(\mathrm{d})$ with occupations close to unity. These account for the biradical character. In addition, the positive overlap between $\pi_{2}$ and $\mathrm{Ti}(\mathrm{d})$ increases as the $\mathrm{Ti}-\mathrm{O}$ distance diminishes when the biradical evolves towards the closed shell configuration through an intersystem crossing as expected for a valence 
tautomerism process. ${ }^{12}$ We can understand both the structure and the reacting pattern of such species by means of the electronic profiles model proposed by Salem. ${ }^{23}$ It includes the three curves represented in Figure 3 to describe the main electronic configurations defining the lowest energy electronic states involved in the valence tautomerism: one curve describes the closed shell configuration $\left(\Psi_{\mathrm{cs}}\right)$ whereas the two other curves describe the open shell or biradical configurations $\left(\Psi_{\mathrm{os}}^{\text {sing }}, \Psi_{\mathrm{os}}^{\text {trip }}\right.$ ), which are all low-lying electronic states of the system with very similar molecular geometries.

$\mathrm{d}(\mathrm{O}-\mathrm{Ti}) \approx 1.8 \AA$

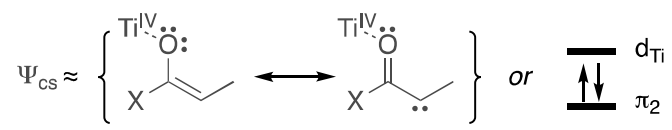

$\mathrm{d}(\mathrm{O}-\mathrm{Ti}) \approx 2.2 \AA$
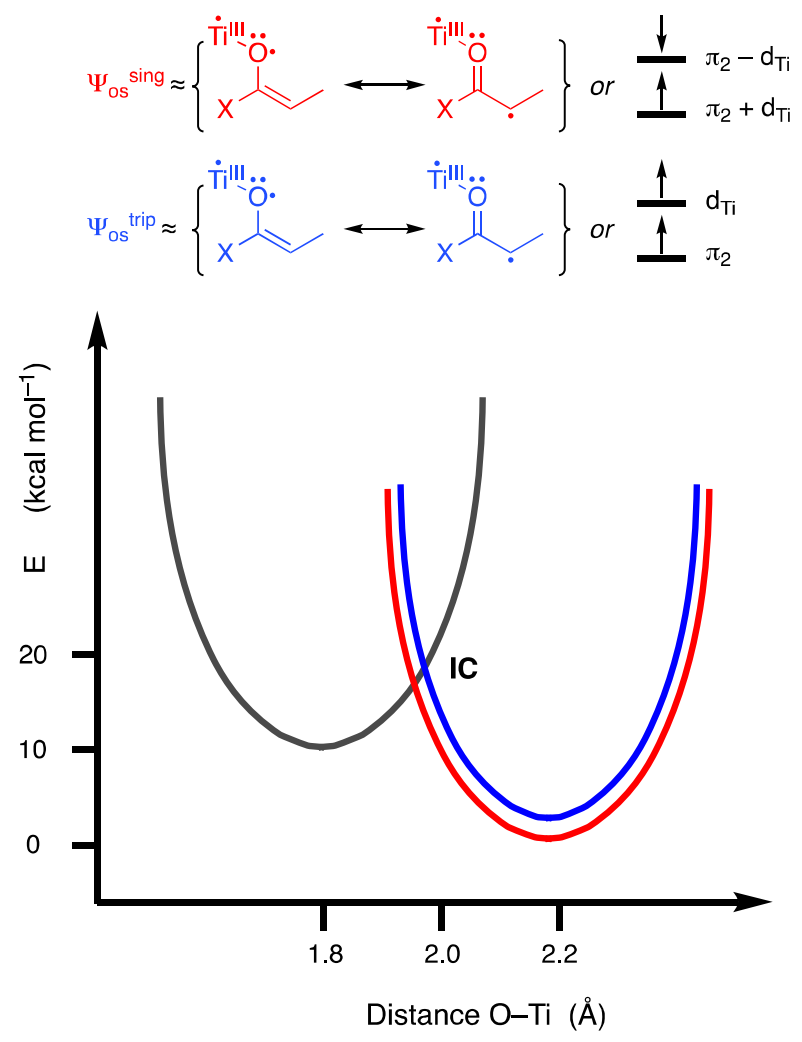

Figure 3. Description of the electronic states involved in the valence tautomerism. 
The energies of the triplet and singlet biradical states, $\Psi_{\mathrm{os}}{ }^{\text {trip }}$ and $\Psi_{\mathrm{os}}{ }^{\text {sing }}$ respectively, are very similar and the states are found to have almost identical geometric and electronic features as can be deduced from the topology of their most significant orbitals shown in Figure 2. In turn, the intersection of the $\Psi_{\mathrm{cs}}$ with the $\Psi_{\mathrm{os}}^{\text {sing }}$ and $\Psi_{\mathrm{os}}{ }^{\text {trip }}$ curves corresponds to an intersystem crossing (IC in Figure 3). In particular, the crossing of $\Psi_{\mathrm{cs}}$ with $\Psi_{\mathrm{os}}^{\text {sing }}$ corresponds to a seam, ${ }^{24}$ and determines the barrier for the interconversion closed shell/biradical species. Noticeably, this valence bond representation of the electronic states is valid in the regions near the corresponding minima and far from the intersection of states where quasidegeneracy induces a strong mixing of electronic states (Figure 3).

The minimum energy structure corresponds to the OSS electronic state for $\mathbf{1}$ because the unfavourable electronic repulsion is compensated by the charge interaction with the titanium atom. In turn, the single occupied orbitals of the biradical structures correspond to a more stable electronic structure than a hypothetical closed shell, because the unfavourable electronrepulsion of the two electrons is not compensated by the titanium charge. The metal is too far from the $\mathrm{C}-\mathrm{C}-\mathrm{O}$ moiety with respect to the closed shell and this is the reason why the unfavourable repulsion is compensated by shortening the Ti-O distance. Indeed, the dual electronic structure obtained by a small change in the $\mathrm{Ti}-\mathrm{O}$ distance characterizes the valence tautomerism of the titanium(IV) enolates.

Comprehensive analysis of such configurations of $\mathbf{1}$ revealed that their geometrical differences do not exceed $0.5 \AA$, at the most (see Figure 2). Nevertheless, the most stable geometry of the CSS configuration features a short Ti-O distance, without single and double bond alternation. Instead, the $\mathrm{O}-\mathrm{C}$ distance becomes shorter and the $\mathrm{C}-\mathrm{C}$ distance longer in the minimum energy structure for both the $\mathrm{T}$ and the OSS radical configurations, which display bond alternation. Most importantly, the energy gap between the closed and open shell configurations decreases as the $\mathrm{Ti}-\mathrm{O}$ distance increases and at $\approx 1.9 \AA$ the biradical 
configurations become more stable than the closed configuration. However, it was not possible to locate the stationary point due to the planarity of the potential energy surface (PES) in this region and the fact that the Born-Oppenheimer approximation is questionable in its proximity due to strong electron-nuclei couplings, making questionable the application of standard optimization methods to explore the non-adiabatic PES. An estimation of the energy barrier for this interconversion at this distance gives a value of the order of $0.2 \mathrm{kcal} \cdot \mathrm{mol}^{-1}$ (see Supporting Information). All together, these calculations indicate that the observed biradical Ti(III) species are originated by subtle changes of the Ti-O distance in a valence tautomery. Interestingly, this valence tautomeric equilibrium is reminiscent of the Peierls distortion observed for extended symmetric systems where competition between open and closed shell electronic states is stabilized by small structural distortions. ${ }^{23}$

The parallel analysis of the sulfur-based model 2 revealed a similar but much more intricate situation, since the larger size of sulfur compared to oxygen modifies the bond distances in the rings that modify the shape of the chiral auxiliary. Thus, the biradical character depends on changes of the Ti-S distance as well as two dihedral angles that affect the whole geometry of the chiral auxiliary. Once again, the new electronic character arises from subtle variations of the geometry of the enolate. In this case, the differences of energy between the minima are lower than those observed in $\mathbf{1}$.

\section{EPR studies on titanium enolates}

The theoretically predicted low energy triplet electronic state, $\Psi_{\mathrm{os}}^{\text {trip }}$, was experimentally identified by EPR studies of the the Ti(III) biradical species coexistent with titanium(IV) enolate derived from 4-benzyl-5,5-dimethyl- $N$-propanoyl-1,3-oxazolidin-2-one used in our synthetic studies (Scheme 2). The EPR spectrum of this enolate at low temperature (Figure 4) 
showed a clear $\left|\Delta \mathrm{m}_{\mathrm{s}}=1\right|$ signal with two superimposed bands centered at $3500 \mathrm{G}$ and $3800 \mathrm{G}$ and with a remarkable half field $\left|\Delta \mathrm{m}_{\mathrm{s}}=2\right|$ fingerprint at $\approx 1580 \mathrm{G}$, which indicates the existence of an electronic triplet state. We assign this signal to the Ti(III) enolate intermediate $\mathrm{T}$ with open shell structure. These spectroscopic data are in close agreement with the computational calculations and all together the results lend a strong support to the coexistence of a biradical intermediate and a closed shell titanium complex that can interconvert through a low energy transition state. ${ }^{25}$ This result is in line with previous studies in similar Ti(IV) complexes in which the EPR signals have been unambiguously assigned based in the observed fine structure and temperature dependence of the spectra. ${ }^{12,13}$

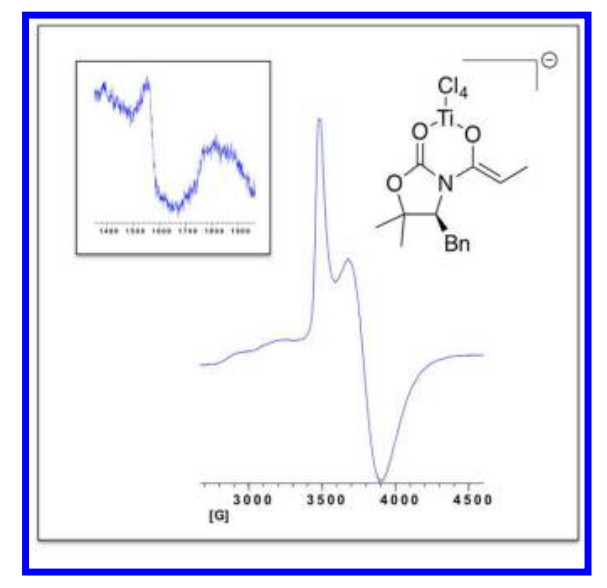

Figure 4. EPR of the Ti(III) biradical species coexistent with the titanium(IV) enolate derived from 4-benzyl5,5-dimethyl- $N$-propanoyl-1,3-oxazolidin-2-one at $5 \mathrm{~K}$ in $\mathrm{CH}_{2} \mathrm{Cl}_{2}$ solution. ${ }^{25}$

The mechanism of the aminoxylation of titanium enolates with TEMPO

Having revealed the electronic structure of the titanium(IV) enolates derived from $N$-acyl oxazolidinones, we next assessed the reaction of $\mathbf{1}$ with a stable radical species such as TEMPO ${ }^{26}$ which might be a model for the mechanistic understanding of the titanium- 
mediated aminoxylation of $N$-acyl-4-benzyl-5,5-dimethyl-1,3-oxazolidin-2-ones developed by some of us. ${ }^{21}$

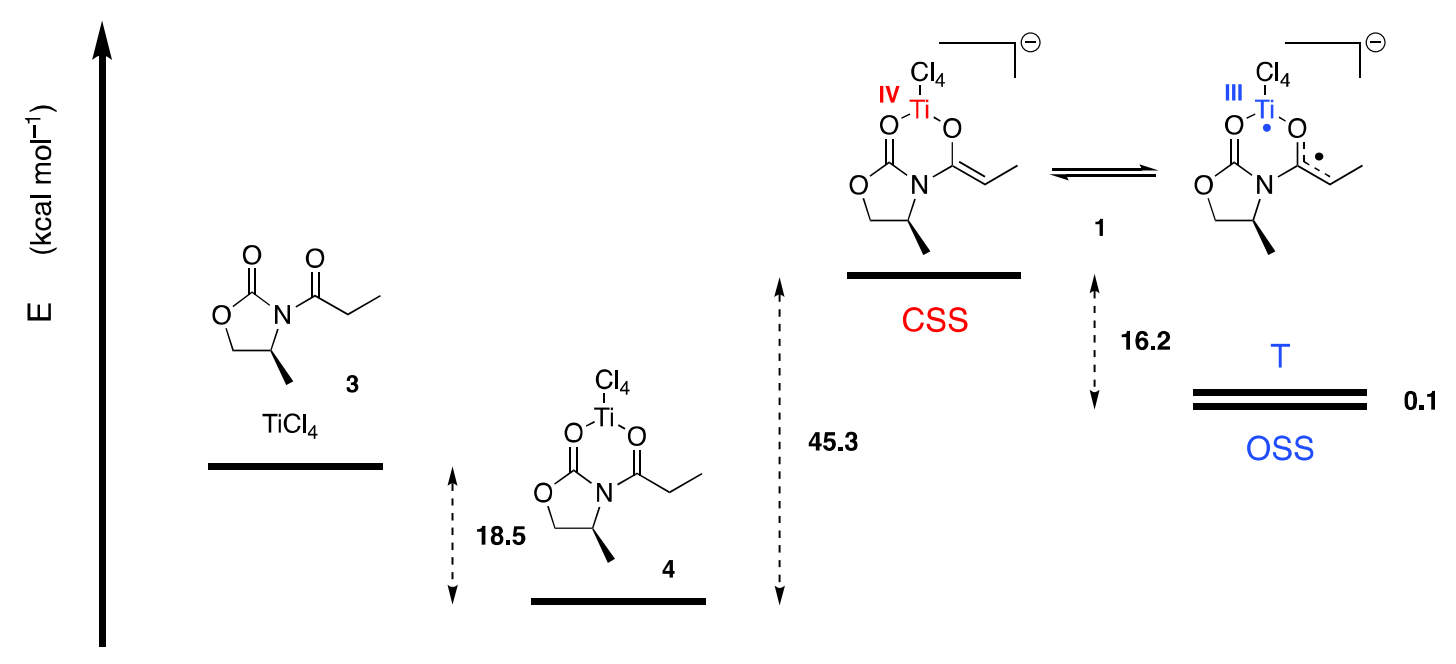

Scheme 3. Reaction profile for the formation of the titanium enolate $\mathbf{1}$ and its valence tautomerism in vacuo using wave-function methods and 6-31G* basis set.

\section{First step: enolization}

The first step of the sequence was the enolization of the parent $N$-propanoyl oxazolidinone 3 with $\mathrm{TiCl}_{4} / \mathrm{Et}_{3} \mathrm{~N} .^{27}$ As anticipated, the interaction of $\mathbf{3}$ with $\mathrm{TiCl}_{4}$ produced a stable chelated intermediate 4 (-18.5 $\mathrm{kcal} \mathrm{mol}^{-1}$, Scheme 3$)$, which was subsequently deprotonated to give the titanium(IV) enolate 1. As shown in Scheme 3, the closed shell singlet configuration (CSS) of 1 turned out to be less stable that the open shell configurations, T and OSS, in about $10 \mathrm{kcal} \mathrm{mol}^{-1}$, which highlights the importance of the biradical component to understand both the structure and the reactivity of titanium enolates. The solvent effects on the energy barriers in vacuo shown in Scheme 3 are expected to largely reduce the energy cost for the proton extraction step since the species involved change their net charge. Indeed, a model of this reaction in $\mathrm{CH}_{2} \mathrm{Cl}_{2}$ solution using the conductor-like polarizable continuum model (C$\mathrm{PCM})^{38,39}$ shows that the energy cost for the proton extraction in the enolization step is largely 
reduced and the CSS Ti(IV) species becomes more stable than 4 by $\sim 20 \mathrm{kcal} \mathrm{mol}^{-1}$. However, the general qualitative shape is maintained, especially in the open shell region of the potential energy surface where the open shell structures are further stabilized (see Supporting Information for details).

\section{Second step: addition of a TEMPO molecule}

With a firmly based description of the structure of enolate 1 to hand, we next examined the second step. This involved the approach of a TEMPO molecule to $\mathbf{1}$ to form the $\mathrm{C}-\mathrm{O}$ bond, which was the crucial step of the aminoxylation reaction. Our calculations revealed that the less sterically hindered $\pi$-face of the biradical configuration of the titanium(IV) enolate, which could be viewed as a formal titanium(III) radical complex, permitted an easy interaction with the TEMPO molecule and the $\mathrm{C}-\mathrm{O}$ bond could be formed in a low barrier transformation that produced the titanium(III) chelate $\mathbf{5}$ (Scheme 4). If the TEMPO molecule approached the enolate from the more sterically hindered $\pi$-face the barrier would be $1-2 \mathrm{kcal}$ $\mathrm{mol}^{-1}$ larger. ${ }^{28}$

Unfortunately, the transition states of these additions are difficult to localize and characterize and the barriers difficult to evaluate due to the size of the molecules and the nature of the radical-radical addition that usually shows a small barrier dominated by steric repulsions. Thus, the differences of energy of the alternative transition states $\left(\Delta \mathrm{E}_{1}\right)$, required to predict the diastereoselectivity ( $5 \boldsymbol{S}$ versus $\mathbf{5} \boldsymbol{R}$ in Scheme 4 ) of the reaction with TEMPO, were estimated from the relative stability of the products $\left(\Delta \mathrm{E}_{2}\right)$ following a thermodynamic approach, which assumes that the differences in energy between transition states are proportional to those in the resultant products. Such an approach is reasonable since the structures of the transition states and the related reactants must be similar because these are $\sim 25 \mathrm{kcal} \mathrm{mol}^{-1}$ higher in energy than the products. 


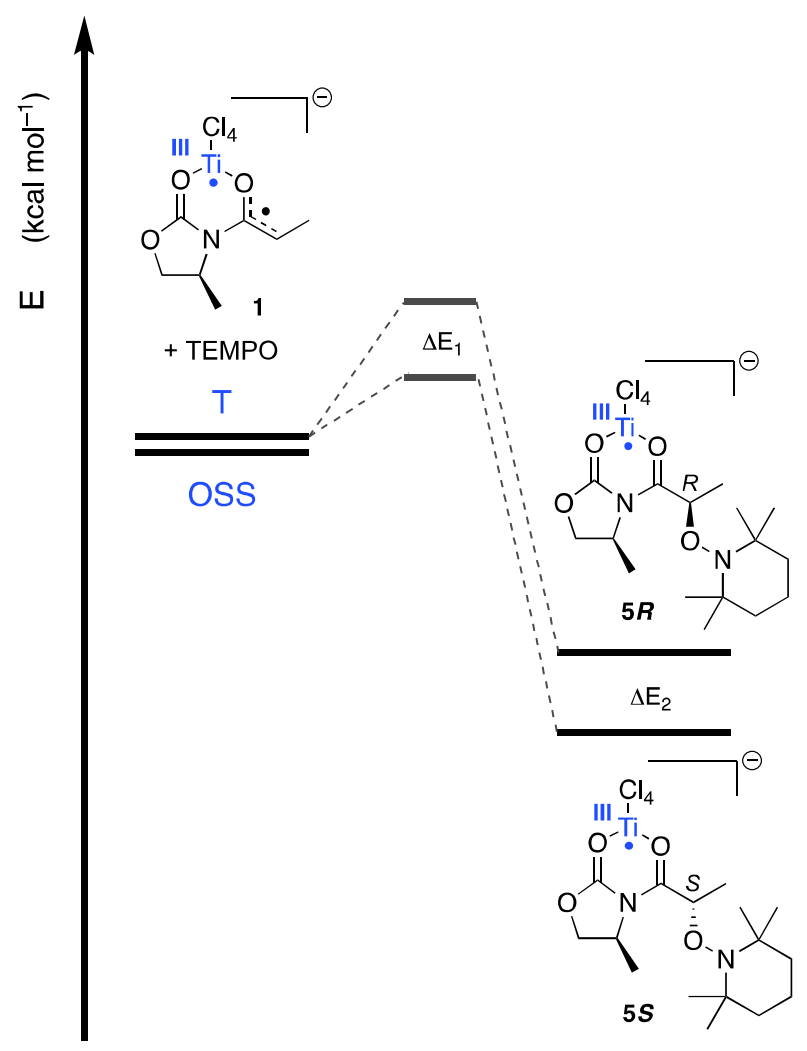

Scheme 4. Reaction profile for the second step at the 6-31G*/UB3LYP level of theory.

\section{Third step: oxidation of titanium(III) chelate 5}

Since the experimental development of the aminoxylation reaction had established the need for two equivalents of TEMPO, we next assessed the role of the second molecule of TEMPO. It was clear from the outset that it should be associated with the significant reducing character of the titanium(III) chelate. This was indeed the case, but the mechanistic description of the process turned out to be troublesome. In fact, the oxidation of the titanium(III) involved a multi-equilibrium process in which the hexacoordinated chelate $\mathbf{5 S}$ possessing two weak dative bonds was transformed into a pentacoordinated Ti(III) complex 6. This was the intermediate that was oxidized by the second TEMPO molecule thereby producing the 
titanium(IV) complex 7, which finally delivered the adduct 8 after the acid work-up (Scheme 5). The oxidized complex is $25-30 \mathrm{kcal} \mathrm{mol}^{-1}$ less stable than the Ti(III)-TEMPO anion radical adduct plus the additional TEMPO molecule as suggested by preliminary calculations using our model in vacuo. This energy difference is expected to be further reduced in a polar solvent that stabilizes large charged molecular species. Noticeably, the proposed mechanism requires much less energy than the direct oxidation of $\mathbf{5 S}$, which makes necessary to take into account the formation of the non-chelated complex $\mathbf{6}$ and the subsequent ligand exchange. ${ }^{13}$

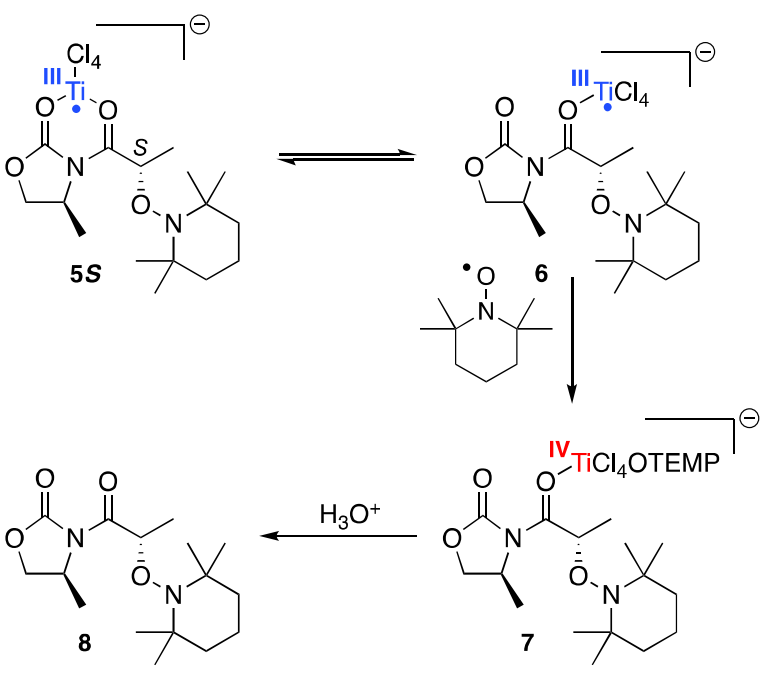

Scheme 5. Third step: oxidation of $\mathbf{5 S}$.

\section{Further analysis}

\section{$\underline{\text { Regioselectivity in conjugate enolates }}$}

The abovementioned mechanistic model was further applied to account for the regioselectivity of the aminoxylation of conjugated enolates. Close inspection of the electronic structure of $\mathbf{1}$ and model conjugated titanium enolates $\mathbf{9}$ and $\mathbf{1 0}$ indicated that the 
coefficients of the SOMO orbitals at the $\alpha, \gamma$, and $\varepsilon$ positions were comparable, whereas those of the $\beta$ and $\delta$ positions were negligible (Figure 5).

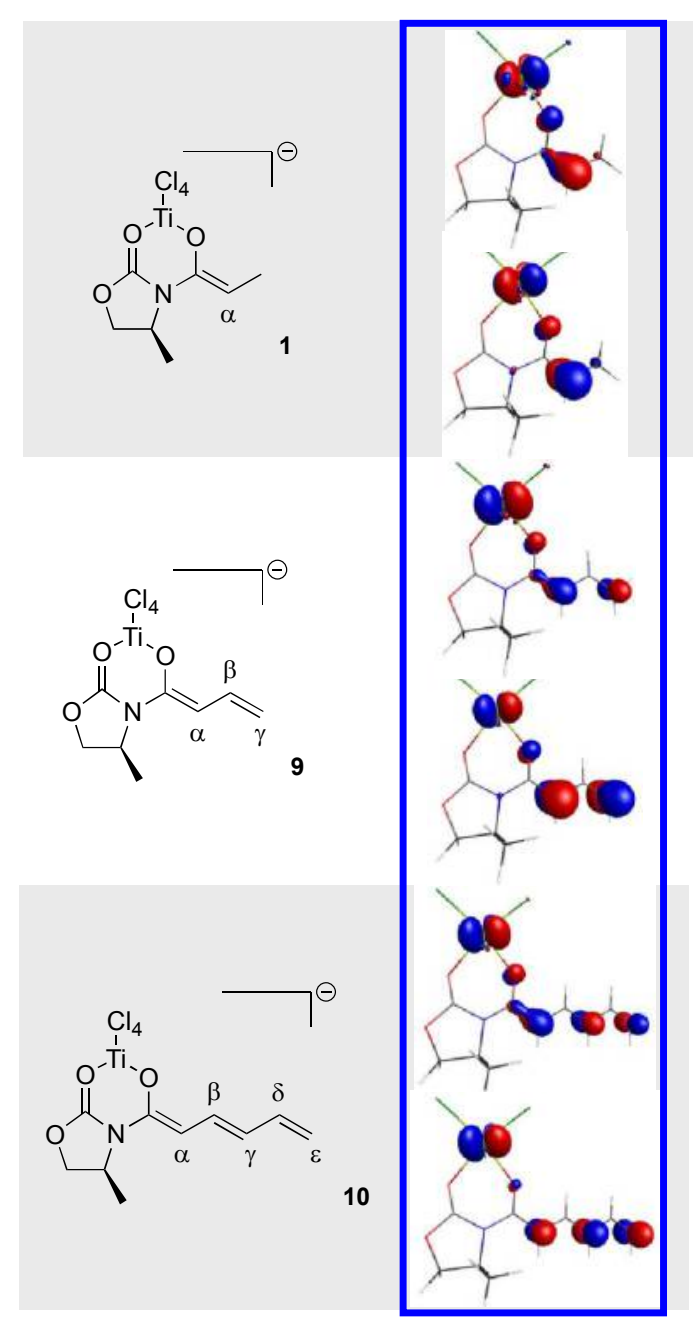

Figure 5. SOMO orbitals of conjugated titanium(IV) enolates taken from monooccupied natural UB3LYP orbitals.

In turn, experimental studies had established that the addition of TEMPO to the titanium(IV) enolates 12a-b derived from $N$-acyl oxazolidinones 11a-b shown in Scheme 6 took place exclusively at the $\gamma$ position; ${ }^{21}$ adducts from the attack to the $\alpha$ or $\beta$ positions were never observed. All together, these results show that the siteselectivity of conjugated enolates is 
primarily based on orbital topology, but also relies on the poorer accessibility of the $\alpha$ position. Unfortunately, the attack of TEMPO to the $\gamma$ position of $\mathbf{1 2 b}(\mathrm{R}: \mathrm{Me})$ afforded a 1:1 diastereomeric mixture of adduct $\mathbf{1 3 b}$, which indicated that the lack of stereocontrol of the addition to the $\gamma$ position was due to the large distance from the chiral centre and the ineffective induction by the chiral auxiliary.

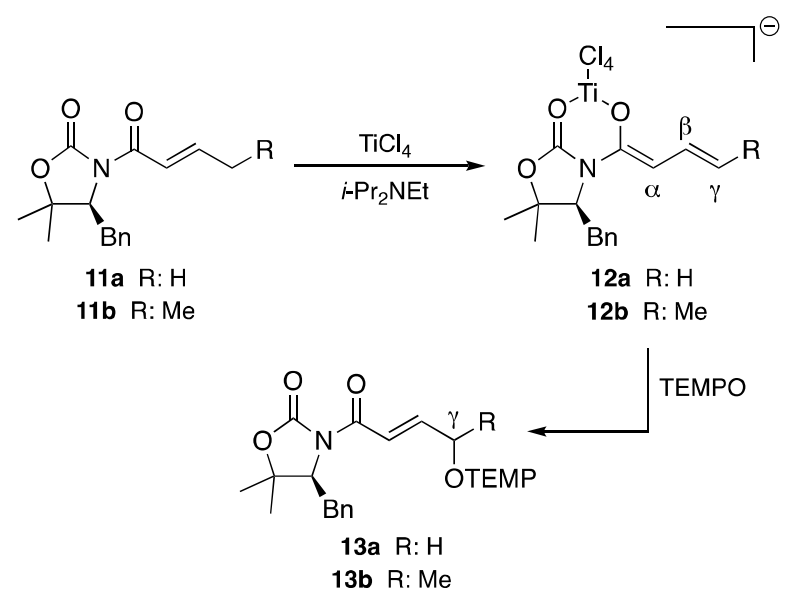

Scheme 6. Siteselective aminoxylation reactions of $\mathbf{1 2}$.

\section{Diastereoselectivity}

Finally, the diastereoselectivities of the TEMPO-aminoxylation of the titanium(IV) enolates derived from several $N$-propanoyl oxazolidinones and thiazolidinethiones 14-16 represented in Scheme 7 were examined using a simple 6-31G*/UB3LYP approach and the diastereomeric ratios were estimated using the formula $\mathrm{P}_{S} / \mathrm{P}_{R}=\exp \left(-\Delta \mathrm{E}_{1} / \mathrm{k}_{\mathrm{B}} \mathrm{T}\right)$ in which $\Delta \mathrm{E}_{1} \approx$ $\Delta \mathrm{E}_{2}$ (see Scheme 4). 


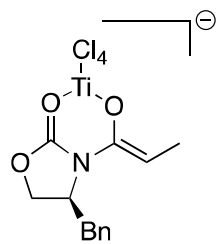

14

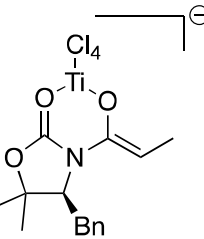

15
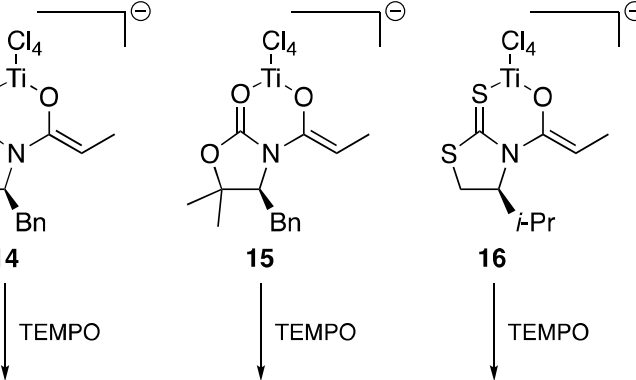

16
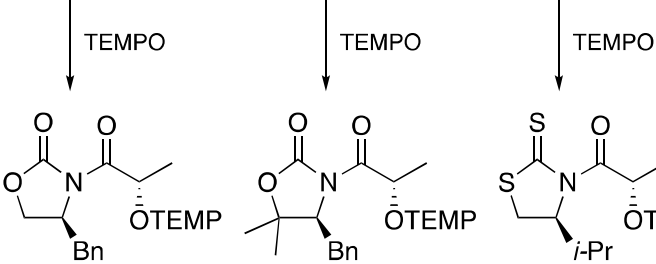

17

Exp dr 78:22

Calcd dr 86:14
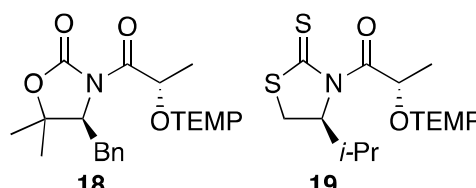

Exp dr 94:

Calcd dr 97:3

Exp dr $91: 9$

Calcd dr 98:2

Scheme 7. Diastereoselective TEMPO-aminoxylation of titanium(IV) enolates from $N$-acyl chiral auxiliaries.

We found that the predicted diastereomeric ratios nicely matched the experimental trends.

This definitely proves the accuracy of the mechanism we report and the validity of our theoretical analysis. Particularly, these calculations indicated that the excellent diastereoselectivity attained with the titanium(IV) enolate from chiral 4-benzyl-5,5-dimethyl$\mathrm{N}$-propanoyl-1,3-oxazolidin-2-one 15, much better than that of the parent oxazolidinone 14, is due to a rigidity of the complex induced by steric repulsion of the geminal methyl groups with the benzyl group that provides a larger exposure of the $\pi$-face $\square$ opposite to the benzyl group. In turn, the distortion produced by the long carbon-sulfur bonds in the thiazolidinethionederived titanium enolate $\mathbf{1 6}$ also facilitates the approach of the electrophile to the opposite face to the isopropyl group and is thus the reason for the high stereocontrol achieved in this case. $^{29}$ 


\section{CONCLUSIONS}

In summary, quantum chemical calculations have established that the appropriate description of the electronic structure of the titanium(IV) enolates derived from oxazolidinones and thiazolidinethiones includes a valence tautomery equilibrium that involves a closed shell and two open shell configurations. As the energy gap between the closed and the open shell configurations is small, subtle changes to the Ti-O distance makes the OSS configuration the most stable with a thermally accessible triplet state $\mathrm{T}$. This reveals the biradical character of these intermediates which are formally $\mathrm{Ti}(\mathrm{III})$ enolates. Such a biradical character has been measured in EPR studies and has been established as the source of radical-like reactivity, which is complementary to the most common nucleophilic profile. In this context, the mechanism of the aminoxylation reaction of titanium(IV) enolates from oxazolidinones with TEMPO has been satisfactorily explained on radical grounds. Importantly, the resulting model accounts for the regio- as well as the diastereoselectivity of such a transformation, which strongly supports the abovementioned calculations. Therefore, both the structure and the reactivity of titanium(IV) enolates, and potentially enolates of other metal, should be examined from this new point of view. Summarizing, the general guideline derived from this study is that titanium(IV) enolates undergo a valence tautomerism equilibrium leading to a coexisting Ti(III) biradical species at normal conditions that should be taken in consideration for a general understanding of their reactivity. Therefore, it should be expected that titanium(IV) enolates can behave either as classical electrophiles or as biradicals depending on the molecule faced by this reagent. One can take advantage of both types of reactivity by choosing an appropriate counterpart and controlling the reaction conditions. 


\section{EXPERIMENTAL SECTION}

\section{Theoretical methods}

All our calculations on the valence tautomerism of titanium(IV) enolates and their reaction with TEMPO were carried out in vacuo employing the standard $6-31 \mathrm{G}^{*}$ basis set and GAMESS 2012 package. $^{30,31}$ The all electron basis set corresponds to that reported by Pople and collaborators: [4s/2s] for $\mathrm{H}$ and $[10 \mathrm{~s} 4 \mathrm{p} 1 \mathrm{~d} / 3 \mathrm{~s} 2 \mathrm{p} 1 \mathrm{~d}]$ for $\mathrm{C}, \mathrm{N}$, and $\mathrm{O} ;^{32}[16 \mathrm{~s} 10 \mathrm{p} 1 \mathrm{~d} / 4 \mathrm{~s} 3 \mathrm{p} 1 \mathrm{~d}]$ for $\mathrm{Cl}$ and $\mathrm{S}^{33}$ and $[22 \mathrm{~s} 16 \mathrm{p} 4 \mathrm{~d} 1 \mathrm{f} / 5 \mathrm{~s} 4 \mathrm{p} 2 \mathrm{~d} 1 \mathrm{f}]$ for $\mathrm{Ti}^{34}$

Regarding the methods used to describe the electronic structure of the intermediates, for the enolization step and valence tautomey process we have used wave function methods (HartreeFock (HF) for closed shell structures and Complete Active Space Self Consistent Field (CASSCF) methods for the multireferential states (CSS, OSS and T) whereas UB3LYP method has been used to describe the intermediate structures involved in the first and second TEMPO additions to understand their regio- and stereoselectivity.

In our studies on the first step of the aminoxylation reaction the structure of the starting oxazolidinone $\mathbf{3}$ and the $\mathrm{TiCl}_{4}$-complex $\mathbf{4}$ were evaluated at RHF level, whereas the structures involved in the valence tautomerism of the titanium enolate were calculated at $\operatorname{CASSCF}(8,7)$ level due to the multireference nature of the electronic states involved. The selection of the $\mathrm{CAS}(m, n)$ was performed using the standard method ${ }^{35}$ based on occupation numbers of the UMP2 natural orbitals for the $\mathbf{T}$ state (those having $1.98<n<0.2$ ) that provided a consistent orbital space for the T, OSS and CSS electronic states beyond the minimum CAS $(4,4)$ space. However, for compound $\mathbf{2}$ it was not possible to define a consistent orbital space beyond CAS $(4,4)$. Nevertheless, the description of these electronic states at CASSCF $(4,4)$ provide molecular structures and energy profile similar to the one shown in Scheme 3. 
Calculations on the chiral TEMPO adducts to obtain $\triangle \mathrm{E}_{2}$ were performed using the UB3LYP approach as implemented in the GAMESS package. This DFT approach allows us to study much more complex systems than the method used in former studies. However, the application of DFT methods based on a single Kohn-Sham determinant to represent the electronic states of open shell systems is questionable, ${ }^{36,37}$ especially for multireference states such as CSS or OSS. Nevertheless, a close inspection of the wave functions of the $\mathbf{T}$ state and the doublet species such TEMPO or the TEMPO adducts can be approximately described by a single determinant with well localized open shell orbitals and, hence, this kind of approximation is justified. The fact that the relevant energy differences in the reaction profiles investigated are due to conformational differences of the organic part the B3LYP functional provides, in principle, a standard, simple and balanced description for a qualitative rationalization of the processes.

Solvent effects on the enolization step of the proposed mechanism have been analyzed by means of the conductor-like polarizable continuum model (C-PCM $)^{38,39}$ implemented in the GAMESS code, ${ }^{30,31}$ using $\mathrm{CH}_{2} \mathrm{Cl}_{2}$ as a solvent. In all cases, zero point energy corrections have been considered.

\section{ASSOCIATED CONTENT}

\section{Supporting Information}

The Supporting Information is available free of charge on the ACS Publications website at http: //pubs.acs.org. 
Supplementary Tables S1-S4 contain Cartesian coordinates of the optimized molecular structures described in the text, Supplementary Figure S1 describes some structure details of the species involved in the valence tautomerism of $\mathbf{1}$ and 2, Supplementary Figure S2 describes the relevant $\operatorname{CASSCF}(4,4)$ natural orbitals of the electronic configurations of $\mathbf{2}$ and the final section Additional calculations describe the results of the calculations performed to investigate the solvent effects on the enolization process of compound $\mathbf{1}$ and an approximate structure of the transition structure between CSS and OSS/T in the IC region of the PES.

\section{AUTHOR INFORMATION}

\section{Corresponding author}

E-mail: i.moreira@ub.edu

Notes

The authors declare no competing financial interests.

\section{ACKNOWLEDGEMENTS}

Financial support from the Spanish Ministerio de Economía y Competitividad and Fondos Feder (Grant Nos. CTQ2012-31034, CTQ2015-65759, and CTQ2016-76423-P) and the Generalitat de Catalunya (2009 SGR825 and 2014 SGR586) as well as a predoctoral studentship to A. G.-P. (APIF, Universitat de Barcelona) are acknowledged.

\section{REFERENCES}


(1) Zabicky, J. The Chemistry of Metal Enolates (Patai's Chemistry of Functional Groups) (Rappoport, Z. Ed.); Wiley-VCH: Chichester, 2009.

(2) Mekelburger, H. B.; Wilcox, C. S. In Comprehensive Organic Synthesis (2 ${ }^{\text {nd }}$ Edition), Vol. 2 (Knochel, P.; Molander, G. A. Eds.); Elsevier, Amsterdam: 2014, pp. 243-272.

(3) Carreira, E. M.; Kvaerno, L. Classics in Stereoselective Synthesis; Wiley-VCH: Weinheim, 2009.

(4) Stoltz, B. M.; Bennett, N. B.; Duquette, D. C; Goldberg, A. F. G.; Liu, Y.; Loewinger, M. M.; Reeves, C. M. In Comprehensive Organic Synthesis (2 ${ }^{\text {nd }}$ Edition), Vol. 3 (Knochel, P. G. Molander, A. Eds.); Elsevier: Amsterdam, 2014, pp. 1-55.

(5) Modern Methods in Stereoselective Aldol Reactions, (R. Mahrwald Ed.); Wiley-VCH: Weinheim, 2013.

(6) For a comprehensive and insightful analysis on the structure and reactivity of lithium enolates, see: (a) Renny, J. S.; Tomasevich, L. L.; Tallmadge, E. H.; Collum, D. B. Angew. Chem. Int. Ed., 2013, 52, 11998-12013. (b) E. H. Tallmadge, D. B. Collum, J. Am. Chem. Soc. 2015, 137, 13087-13095.

(7) For a representative example, see Yu, K.; Lu, P.; Jackson, J. J.; Nguyen, T.-A. D.; Alvarado, J.; Stivala, C. E.; Ma, Y.; Mack, K. A.; Hayton, T. W.; Collum, D. B.; Zakarian, A. J. Am. Chem. Soc. 2017, 139, 527-533.

(8) Ligands are said to be innocent when they allow oxidation states of the central atoms to be well defined and permanent. See: Jørgensen, C. K. Coordin. Chem. Rev., 1966, 1, 164178.

(9) (a) de Bruin, B. Eur. J. Inorg. Chem., 2012, 340-342. (b) Luca, O. R.; Crabtree, R. H. Chem. Soc. Rev., 2013, 42, 1440-1459.

(10) For the role of non-innocent ligands in catalysis, see: (a) Chirik, P. J.; Wieghardt, K. Science, 2010, 327, 794-795. (b) Dzik, W. I.; van der Vlugt, J. I.; Reek, J. N. H.; de Bruin, B. 
Angew. Chem. Int. Ed., 2011, 50, 3356-3358. (c) Lyaskovskyy, V.; de Bruin, B. ACS Catal., 2012, 2, 270-279.

(a) Evangelio, E.; Ruiz-Molina, D. Eur. J. Inorg. Chem., 2005, 2957-2971. (b) Tezgerevska, T.; Alley, K. G.; Boskovic, C. Coordin. Chem. Rev., 2014, 268, 23-40.

(12) Moreira, I de P. R.; Bofill, J. M.; Anglada, J. M.; Solsona, J. G.; Nebot, J.; Romea, P.; Urpí, F. J. Am. Chem. Soc., 2008, 130, 3242-3243.

(13) Heras, C.; Ramos-Tomillero, I.; Caballero, M.; Paradís-Bas, M.; Nicolás, E.; Albericio, F.; Moreira, I. de P. R.; Bofill, J. M. Eur. J. Org. Chem., 2015, 2111-2118.

(14) For a recent overview on the titanium(IV) enolate chemistry, see: Ciez, D.; Palasz, A.; Trzewik, B. Eur. J. Org. Chem., 2016, 1476-1493.

(15) (a) Kise, N.; Ueda, T.; Kumada, K.; Terao, Y.; Ueda, N. J. Org. Chem., 2000, 65, 464-468. (b) Nguyen, P. Q.; Schäfer, H. J. Org. Lett., 2001, 3, 2993-2995.

(16) Csákÿ, A. G.; Plumet, J. Chem. Soc. Rev. 2001, 30, 313-320.

(17) Richter, J. M.; Whitefield, B. W.; Maimone, T. J.; Lin, D. W.; Castroviejo, M. P.; Baran, P. S. J. Am. Chem. Soc., 2007, 129, 12857-12869.

(18) (a) Beaumont, S.; Ilardi, E. A.; Monroe, L. R.; Zakarian, A. J. Am. Chem. Soc., 2010, 132, 1482-1483. (b) Herrmann, A. T.; Smith, L. L.; Zakarian, A. J. Am. Chem. Soc., 2012, 134, 6976-6979. (c) Gu, Z.; Herrmann, A. T.; Zakarian, A. Angew. Chem. Int. Ed., 2011, 50, $7136-7139$.

(19) For an overview, see Amatov, T.; Jahn, U. Angew. Chem. Int. Ed., 2011, 50, 45424544.

(20) Mabe, P. J.; Zakarian, A. Org. Lett., 2014, 16, 516-519.

(21) Gómez-Palomino, A.; Pellicena, M.; Romo, J. M.; Solà, R.; Romea, P.; Urpí, F.; FontBardia, M. Chem. Eur. J., 2014, 20, 10153-10159. 
(22) In fact, enolates $\mathbf{1}$ and $\mathbf{2}$ are ate complexes. The putative titanium(IV) enolates should contain three chlorine atoms bound to the titanium metal without a net charge. However, such species have been calculated to be much less stable than the aforementioned ate complexes.

(23) Salem, L. In The Molecular Orbital Theory of Conjugated Systems; W. A. Benjamin: New York, 1966, pp. 495-505.

(24) The potential energy surfaces of different spin symmetry can intersect in a hyperline called seam. The two potential energy surfaces, each one corresponding to singlet states, lead to a conical intersection or a weakly avoided crossing depending on the nature of the electronic states involved. See Schlegel H. B. Modern Electronic Structure Theory, Part I (Yarkony, D. R., Ed.); World Scientific: Singapore, 1995.

(25) The EPR spectra of the titanium enolates (X-band, $\square \square \square \square 9.414185 \mathrm{GHz}$ ) have been recorded at $5 \mathrm{~K}$ in $\mathrm{CH}_{2} \mathrm{Cl}_{2}$ solution after reacting the corresponding ketone with $\mathrm{TiCl}_{4}$ and triethylamine under inert atmosphere. Standard blank ground line correction has been applied and do not show any signal in the explored regions. Consequently these signals are unambiguously assigned to the $\mathrm{Ti}(\mathrm{III})$ enolate intermediate $\mathrm{T}$ with open shell structure. Similar studies were carried out on sulfur-based analogs. Unfortunately, these compounds produce EPR signals of much lower intensity even at low temperature. This observation can be expected since the OSS and T biradical states of these derivatives are predicted to be closer in energy to the CSS state $\left(5.8 \mathrm{kcal} \mathrm{mol}^{-1}\right)$.

(26) For the use of TEMPO and other nitroxides in synthesis, see: (a) Vogler, T.; Studer, A. Synthesis, 2008, 1979-1993. (b) Tebben, L.; Studer, A. Angew. Chem. Int. Ed., 2011, 50, 5034-5068.

(27) Evans, D. A.; Urpí, F.; Somers, T. C.; Clark, J. S.; Bilodeau, M. T. J. Am. Chem. Soc., 1990, 112, 8215-8216. 
(28) The triplet state of the enolate has been used to calculate the approach to the TEMPO molecule, which gives the adduct in the doublet electronic state.

(29) For the influence of chiral auxiliaries on stereoselective reactions from titanium enolates, see Baiget, J.; Cosp, A.; Gálvez, E.; Gómez-Pinal, L.; Romea, P.; Urpí, F. Tetrahedron, 2008, 64, 5637-5644.

(30) Schmidt, M. W.; Baldridge, K. K.; Boatz, J. A.; Elbert, S. T.; Gordon, M. S.; Jensen, J. H.; Koseki, S.; Matsunaga, N.; Nguyen, K. A.; Su, S.; Windus, T. L.; Dupuis, M.; Montgomery, J. A., Jr. J. Comput. Chem., 1993, 14, 1347-1363.

(31) Gordon, M. S.; Schmidt, M. W. In Theory and Applications of Computational Chemistry, the first forty years (Dykstra, C. E.; Frenking, G.; Kim, K. S.; Scuseria, G. E., Eds), Chapter 41, pp. 1167-1189. Elsevier: Amsterdam, 2005.

(32) Hariharan, P. C.; Pople, J. A. Theoret. Chimica Acta, 1973, 28, 213-222.

(33) Francl, M. M.; Petro, W. J.; Hehre, W. J.; Binkley, J. S.; Gordon, M. S.; DeFrees, D. J.; Pople, J. A. J. Chem. Phys., 1982, 77, 3654-3665.

(34) Rassolov, V.; Pople, J. A.; Ratner, M.; Windus, T. L. J. Chem. Phys., 1998, 109, $1223-1229$.

(35) Bofill, J. M.; Pulay, P. J. Chem. Phys., 1989, 90, 3637-3646.

(36) Caballero, M.; Moreira, I. de P. R.; Bofill, J. M. J. Chem. Phys., 2013, 138, 174107. Mol. Phys. 112, 809 (2013) and J. Chem. Phys. 138, 174107 (2013)

(37) Caballero, M.; Moreira, I. de P. R.; Bofill, J. M. Mol. Phys., 2013, 112, 809-817.

(38) Barone, V.; Cossi, M. J. Phys. Chem. A, 1998, 102, 1995-2001.

(39) Cossi, M.; Rega, N.; Scalmani, G.; Barone, V. J. Comput. Chem. 2003, 24, 669-681. 